\title{
Geometric Design of Eight-Bar Wearable Devices based on Limb Physiological Contact Task
}

\author{
Nina Robson ${ }^{\mathrm{a}}$, Gim Song Soh ${ }^{\mathrm{b}, *}$ \\ ${ }^{a}$ Department of Mechanical Engineering, California State University, Fullerton, California \\ 92831 \\ ${ }^{b}$ Engineering Product Development, Singapore University of Technology and Design, \\ Singapore 487372
}

\begin{abstract}
This paper describes a geometric design method for the motion generation of mechanical wearable devices based on desired limb physiological contact task, parametrized by first and second order motion specifications. Specifically, our approach combines the higher order motion task specifications with anthropometric back-bone chain to synthesize planar eight-bar linkages that achieve the desired motion task. The method is demonstrated by an example that offers a novel alternative approach for wearable devices design: a comprehensive systematic process to create devices, based on a backbone chain that is sized according to the physical dimensions of the human limb. Once it is ensured that the backbone chain motion is close to the physiological one, the rest of the multi-loop wearable device is designed. In the end of the process the wearable device is attached, so that the backbone chain parallels the human's limb itself to provide the skeletal structure for the limb yet passively follow its motion. This results in mechanical devices with compact size, minimal actuation and better wearability.
\end{abstract}

Keywords: motion generation, eight-bar linkage, wearable robot

\footnotetext{
* Corresponding author

Email addresses: nrobson@fullerton.edu (Nina Robson), sohgimsong@sutd.edu.sg (Gim Song Soh)
}

Preprint submitted to Elsevier

February 24, 2016

(C) 2016. This manuscript version is made available under the Elsevier user license http://www.elsevier.com/open-access/userlicense/1.0/ 


\section{Introduction}

Recently, there has been a need to reduce the number of actuators within robotic limbs, so as to provide better reliability and lower cost. This was achieved by coupling the motion of multiple joints, leading to designs with fewer actuators than its degrees-of-freedom. Such limbs termed "under-actuated" or "minimally-actuated" has demonstrated significant benefits especially in grasping applications due to their passive adaptability nature between the degrees-offreedom, thus allowing the fingers to conform to the environment shape without the need for sensing.

There are generally two types of under-actuated robotic limbs proposed in literature. The first type is based on tendon-actuated systems and the second type is based on mechanical linkages. A tendon driven robotic hand can be usually designed with compact size and dexterous operation. However, such systems suffer from friction and elasticity issues during operation and are limited to small grasping forces [1]. On the other hand, linkage mechanisms are preferable for applications where high stability and large forces are expected. Gosselin et al. [2] designed a flexible and versatile linkage based mechanical gripper for industry applications. The TUAT/Karlsruhe Hand [3] and mechanical finger [4] are driven by single degree of freedom linkages meant for prosthetics applications.

Some of the current developments in the field of wearable devices and exoskeletons in particular, focus on restoring abilities lost due to injury or compensating for persistent disabilities. For example, exoskeletons have been developed for rehabilitation after neurologic injury such as stroke or traumatic brain injury. These robotic devices have been developed for a variety of tasks, including gait rehabilitation (such as $[5,6])$ and upper extremity rehabilitation (see $[7,8]$ for reviews). For hand rehabilitation, Wang et al. [9] designed an exoskeleton based on 4 bar linkage for an index finger rehabilitation and Yihun et al. [10] recently designed a non-anthropomorphic wearable device based on eightbar linkage for thumb rehabilitation. Wearable devices and exoskeletons have 
also been designed and developed for the purpose of augmenting and amplifying the ability of humans (for reviews see $[11,12]$ ). Human augmenting and amplifying exoskeletons have been developed for both military and industrial applications. Examples include leg exoskeletons for hauling heavy loads [13] and upper-extremity robots for strength augmentation [14].

The most important requirement for the design of wearable devices is safety and is usually achieved through some form of mechanical range stopper or the design itself [15]. The key approach is to design the wearable device with its rotation axis coinciding with that of the human joint so as to mimic its workspace. This way, even though there is a failure on the device controller, the exoskeleton will not force the user to move in an unnatural manner resulting in damage to his limbs. There are a few ways in which one could achieve this. The most direct manner is to match the joint centers directly [16]. However, this approach requires structural space on the side of the limbs. Alternatively, a remote center of rotation structure can be considered $[17,18]$.

The requirement of having both the limb and wearable device to coincide can be disregarded if flexible or underactuated structures are used. An approach of making it flexible is to adopt a linkage with redundant degrees of freedom [19]. Another way is through tendon-driven mechanisms [20,21] or soft pneumatic actuators as part of a wearable system [22]. Underactuated wearable devices are usually achieved by attaching and controlling the movement of the distal segment directly [23].

It is important to note, that while most of the above mentioned devices show satisfactory performance, there still does not exist a systematic methodology for the design of these systems. The aforementioned highlights the need for the development of novel design techniques for underactuated linkage skeletal structures that are sized according to the wearers limb dimensions. Our original intent is to seek inspiration from nature, as similar to many insects or animals, where the exoskeleton supports or protects an animals body and is shaped according to the shape and size of a particular insect or animal. In this paper we extend upon our initial work in Robson et al. [24] and show that the ability 
to first identify the desired human motions and then mathematically describe them as physiological task, using higher order motion specifications, opens the possibility of creating mechanical limbs with minimal number of actuators and better wearability. Here, we would like to note that unlike other wearable device design techniques that use parallel mechanical linkages [25, 26, 27], we offer a novel alternative approach: a comprehensive systematic process to create wearable device that incorporate anthropometric backbone chain with the physical dimensions of the human limb and a physiological task, compatible with the contact constraints between the limb and objects or the environment. Since the backbone chain provides the skeletal structure of the whole system, once it is ensured that its motion is close to the physiological task, then the rest of the multi-loop kinematic system is designed. In the end of the process, the wearable device is attached, such that the backbone chain braces its human counter part. This approach is particularly useful for synthesizing mechanisms that require physiological contact task during motion such as walking or grasping.

\section{Physiological Contact Task Identification, using Sensor Data}

Our goal is to design mechanical linkage skeletal structures, based on anthropometric data from a human limb that can be easily attached, avoiding collision between the linkage and the wearer's limb, yet incorporating higher order kinematic constraints for contact tasks such as walking or grasping. In particular, for this paper, we chose the design of an index finger of a wearable grasping device as an example to illustrate the usefulness of this approach. Grasping devices are tools that are used for manipulation and handling tasks, which require the $\operatorname{limb}(\mathrm{s})$ to be in contact with an object. The paper is inspired by Howard and Kumar [28], who show that the stability of a grasp depends not only on the magnitude and direction of the contact forces but also on the local curvature of the contacting bodies. Another inspiration for the current paper are the works

of Rimon and Burdick [29, 30] who analyze the mobility of bodies in contact to show that first order theories based on positions and velocities are not sufficient 
to define the local behavior of a linkage mechanism. The authors take a step further to show that the acceleration properties of movement are related to the curvature of the contacting bodies and can be used to effectively constrain a rigid body for grasping applications. In order to define the contact requirements for each finger, our research builds upon the above-mentioned works, by incorporating velocity and acceleration task requirements into our synthesis procedure.

Our design approach starts by obtaining experimental data of subject(s), performing desired grasping task(s), using 3D motion capture system. In order to identify the human motion and its derivatives Robson et al. [31] has been working on inertial measurements combined with the finger motion capture data obtained from a Vicon motion capture system. For example in [32], the authors develop a cost-effective glove prototype to obtain position and higher order derivative information of each fingertip (see Figure 1). The sensor-glove device

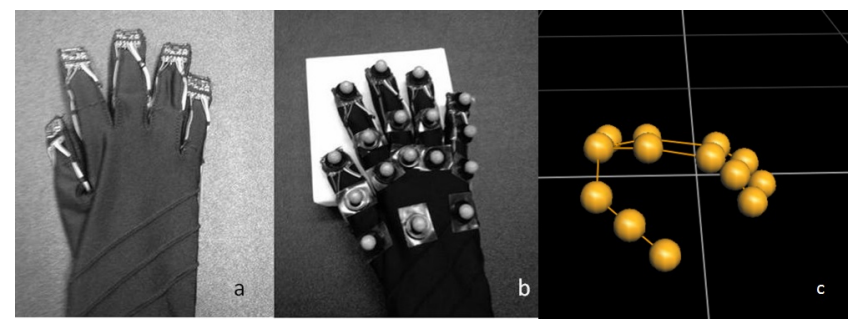

Figure 1: The sensor based glove, developed for extracting higher order motion data from human's hand.

consists of tri-axis accelerometers located at the fingertips and optical markers placed in accordance with a reduced marker protocol. The information from the accelerometers and the markers is used to obtain human finger motion data based on position, velocity and acceleration. The latter is then used to define the physiological contact task for the synthesis of the single degree-of-freedom multiloop kinematic chain. For example, we chose a set of two task specifications such that $P V_{\text {start }}$ defines the start position and velocity and $P V A_{\text {end }}$ defines the end position, velocity and acceleration of the limb motion. The acceleration 
is typically specified in positions where not only contact but curvature effects, between the finger and an object with a known geometry, are taken into account.

Once the human motions are recorded and the contact task is specified, linkage based mechanism can be designed for the chosen physiological task. To account for the higher order motion task constraints, a modification of the eight-bar linkage design process described in [33] is developed. An eight-bar mechanism is chosen to be synthesized due to the design freedom on the choice of ground pivots. This property allows for packaging the linkage into a compact size in order to be used as a wearable device.

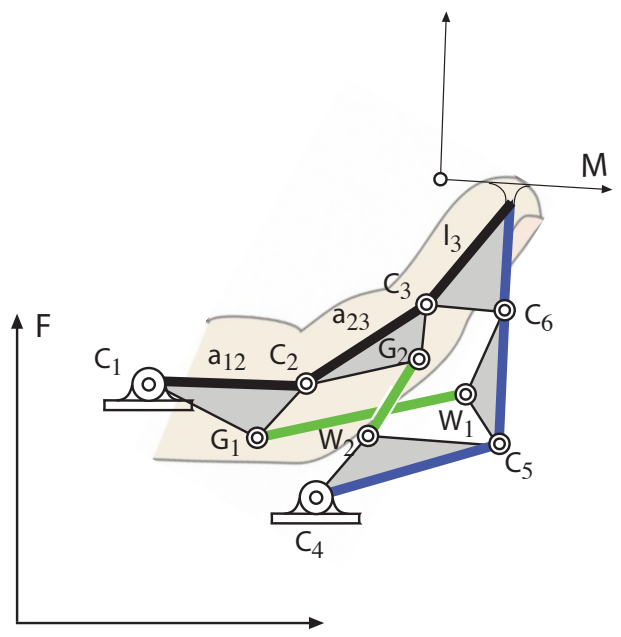

Figure 2: The linkage-based finger device.

\section{Method For Designing Minimally Actuated Mechanical Linkages Based on the Identified Workspace}

Our geometric design methodology consists of 3 steps. First, we specify the various link dimensions of a parallel $3 \mathrm{R}$ chain $\mathbf{C}_{1}-\mathbf{C}_{6}$ "backbone" chain as shown in Figure 2, such that $a_{12}, a_{23}$ and $l_{3}$ are based on human limb dimensions and the resulting robot moves through the set of specified poses. Next, we solve for the robot joint parameters at each of the specified poses to determine the 
positions, velocity and acceleration of the various links at each of the specified poses. These matrices then yield the basis for the geometric design of two RR chains $\mathbf{G}_{1} \mathbf{W}_{1}$ and $\mathbf{G}_{2} \mathbf{W}_{2}$ to form an eight-bar linkage based wearable device.

The synthesis of an RR link to reach a set of $P_{1} V_{1}$ and $P_{2} V_{2} A_{2}$ positions can be found by solving the following set of design equations,

$$
\begin{gathered}
\left(\mathbf{W}^{2}-\mathbf{G}^{2}\right) \cdot\left(\mathbf{W}^{2}-\mathbf{G}^{2}\right)-\left(\mathbf{W}^{1}-\mathbf{G}^{1}\right) \cdot\left(\mathbf{W}^{1}-\mathbf{G}^{1}\right)=0, \\
\left(\left[\Omega_{k, 1}\right] \mathbf{W}^{1}-\left[\Omega_{l, 1}\right] \mathbf{G}^{1}\right) \cdot\left(\mathbf{W}^{1}-\mathbf{G}^{1}\right)=0, \\
\left(\left[\Omega_{k, 2}\right] \mathbf{W}^{2}-\left[\Omega_{l, 2}\right] \mathbf{G}^{2}\right) \cdot\left(\mathbf{W}^{2}-\mathbf{G}^{2}\right)=0, \\
\left(\left[\Lambda_{k, 1}\right] \mathbf{W}^{1}-\left[\Lambda_{l, 1}\right] \mathbf{G}^{1}\right) \cdot\left(\mathbf{W}^{1}-\mathbf{G}^{1}\right)+\left(\left[\Omega_{k, 1}\right] \mathbf{W}^{1}-\left[\Omega_{l, 1}\right] \mathbf{G}^{1}\right) . \\
\left(\left[\Omega_{k, 1}\right] \mathbf{W}^{1}-\left[\Omega_{l, 1}\right] \mathbf{G}^{1}\right)=0,
\end{gathered}
$$

where $\left[\Omega_{k, j}\right]$ denote the velocity of the $k^{t h}$ moving link, and $\left[\Omega_{l, j}\right]$ and $\left[\Lambda_{l, j}\right]$ denote the velocity and acceleration of the $l^{\text {th }}$ moving link at its $j^{\text {th }}$ position with

$$
\begin{aligned}
& {\left[B_{1 j}\right]=[G]\left[Z\left(\theta_{1}\right)\right]\left[X\left(a_{12}\right)\right],} \\
& {\left[B_{2 j}\right]=\left[B_{1 j}\right]\left[Z\left(\theta_{2}\right)\right]\left[X\left(a_{23}\right)\right],} \\
& {\left[B_{3 j}\right]=\left[B_{2 j}\right]\left[Z\left(\theta_{3}\right)\right][H],} \\
& {\left[\Omega_{1 j}\right]=\left[\dot{B_{1 j}}\right]\left[B_{1 j}\right]^{-1},} \\
& {\left[\Omega_{2 j}\right]=\left[\dot{B_{2 j}}\right]\left[B_{2 j}\right]^{-1}} \\
& {\left[\Omega_{3 j}\right]=\left[\dot{B_{3 j}}\right]\left[B_{3 j}\right]^{-1},} \\
& {\left[\Lambda_{1 j}\right]=\left[\ddot{B_{1 j}}\right]\left[B_{1 j}\right]^{-1},} \\
& {\left[\Lambda_{2 j}\right]=\left[\ddot{B_{2 j}}\right]\left[B_{2 j}\right]^{-1},} \\
& {\left[\Lambda_{3 j}\right]=\left[\ddot{B_{3 j}}\right]\left[B_{3 j}\right]^{-1}, \quad j=1,2 .}
\end{aligned}
$$

The coordinate transformation $[G]$ defines the position of the base link with origin at $C_{1}$, and the coordinate transformation $[H]$ locates the task frame $M$ relative to the end-effector frame $B_{3}$. The matrices $[Z(\theta)]$ and $[X(a)]$ are homogenous transformations that rotate a body attached frame about its z-axis. 
The fixed $\mathbf{G}^{2}$ and moving $\mathbf{W}^{2}$ pivots of its $k^{\text {th }}$ and $l^{\text {th }}$ moving link at the $2^{\text {nd }}$ position are related to its first position by,

$$
\mathbf{G}^{2}=\left[B_{k, 2}\right]\left[B_{k, 1}\right]^{-1} \mathbf{G}^{1} \quad \text { and } \quad \mathbf{W}^{2}=\left[B_{l, 2}\right]\left[B_{l, 1}\right]^{-1} \mathbf{W}^{1}
$$
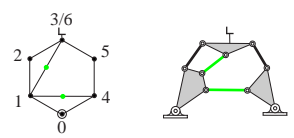

- Independent
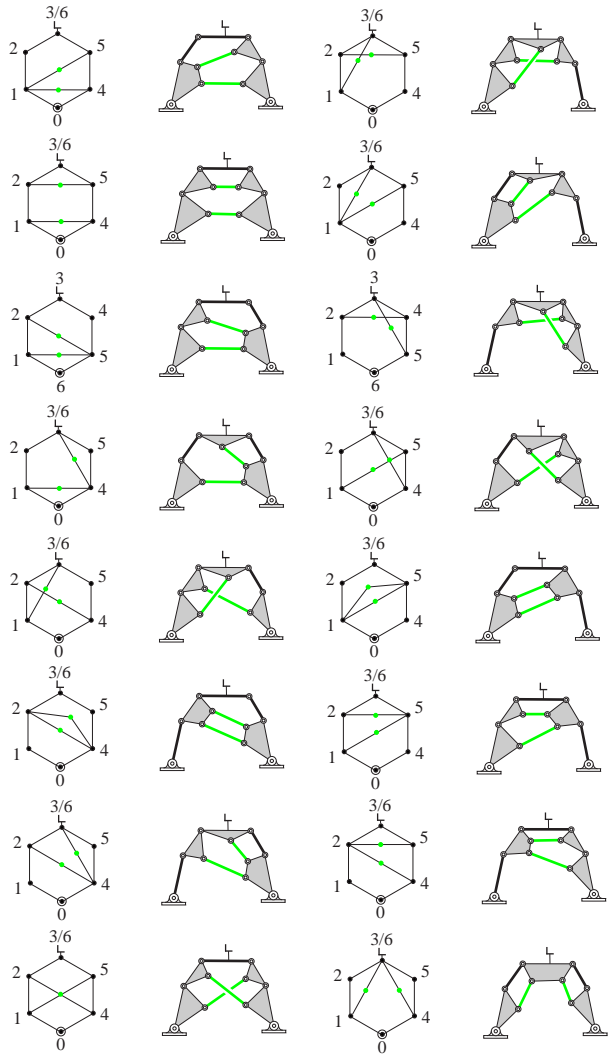

Figure 3: The 17 different ways in which two RR links can be added to a parallel $3 \mathrm{R}$ chain independently to yield a one degree-of-freedom eight bar linkage.

Figure 3 shows the seventeen various ways of attaching two $R R$ links if the designer chooses not to constrain a link to ground or to the new link formed by the first RR chain. For more details, refer to McCarthy and Soh (2010) [33]. 


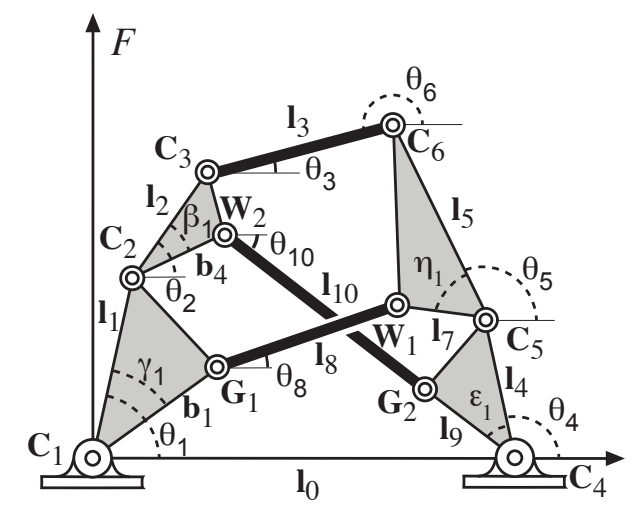

Figure 4: The joint angles and link parameters for the eight-bar linkage.

\subsection{Analysis}

To analyze the resulting eight-bar linkage, we first formulate the loop equations using complex number coordinates and solve using the Dixon determinant [36]. Consider the eight-bar linkage as shown in Figure 4. Introduce a coordinate frame with $\mathbf{C}_{\mathbf{1}}$ as the origin and its x-axis directed toward $\mathbf{C}_{4}$. The vector loop equations formed by $\mathbf{C}_{1} \mathbf{G}_{1} \mathbf{W}_{1} \mathbf{C}_{5} \mathbf{C}_{4}, \mathbf{C}_{1} \mathbf{C}_{2} \mathbf{W}_{2} \mathbf{G}_{2} \mathbf{C}_{4}$ and $\mathbf{C}_{1} \mathbf{C}_{2} \mathbf{C}_{3} \mathbf{C}_{6} \mathbf{C}_{5} \mathbf{C}_{4}$, can be written in complex form as

$$
\begin{array}{ll}
\mathcal{C}_{1}: & b_{1} \Theta_{1} e^{-i \gamma_{1}}+l_{8} \Theta_{8}-l_{4} \Theta_{4}-l_{7} \Theta_{5} e^{i \eta_{1}}-l_{0}=0, \\
\mathcal{C}_{2}: & l_{1} \Theta_{1}+b_{4} \Theta_{2} e^{-i \beta_{1}}+l_{10} \Theta_{10}-l_{9} \Theta_{4} e^{i \epsilon_{1}}-l_{0}=0, \\
\mathcal{C}_{3}: & l_{1} \Theta_{1}+l_{2} \Theta_{2}+l_{3} \Theta_{3}-l_{4} \Theta_{4}-l_{5} \Theta_{5}-l_{0}=0,
\end{array}
$$

with complex conjugate

$$
\begin{array}{ll}
\mathcal{C}_{1}^{\star}: & b_{1} \Theta_{1}^{-1} e^{i \gamma_{1}}+l_{8} \Theta_{8}^{-1}-l_{4} \Theta_{4}^{-1}-l_{7} \Theta_{5}^{-1} e^{-i \eta_{1}}-l_{0}=0, \\
\mathcal{C}_{2}^{\star}: & l_{1} \Theta_{1}^{-1}+b_{4} \Theta_{2}^{-1} e^{i \beta_{1}}+l_{10} \Theta_{10}^{-1}-l_{9} \Theta_{4}^{-1} e^{-i \epsilon_{1}}-l_{0}=0, \\
\mathcal{C}_{3}^{\star}: & l_{1} \Theta_{1}^{-1}+l_{2} \Theta_{2}^{-1}+l_{3} \Theta_{3}^{-1}-l_{4} \Theta_{4}^{-1}-l_{5} \Theta_{5}^{-1}-l_{0}=0,
\end{array}
$$

where $\Theta_{i}=e^{i \theta_{i}}$. This set of equations can be solved easily using the Dixon determinant for $\Theta_{i}, i=2,3,4,5,8,10$ for a given input angle $\Theta_{1}$. 
Next, to determine the joint rates, we compute the derivative of the loop equations (4) and (5), to obtain

$$
\begin{array}{ll}
\nabla \mathcal{C}_{1}: & b_{1} \dot{\Theta}_{1} e^{-i \gamma_{1}}+l_{8} \dot{\Theta}_{8}-l_{4} \dot{\Theta}_{4}-l_{7} \dot{\Theta}_{5} e^{i \eta_{1}}=0, \\
\nabla \mathcal{C}_{2}: & l_{1} \dot{\Theta}_{1}+b_{4} \dot{\Theta}_{2} e^{-i \beta_{1}}+l_{10} \dot{\Theta}_{10}-l_{9} \dot{\Theta}_{4} e^{i \epsilon_{1}}=0, \\
\nabla \mathcal{C}_{3}: & l_{1} \dot{\Theta}_{1}+l_{2} \dot{\Theta}_{2}+l_{3} \dot{\Theta}_{3}-l_{4} \dot{\Theta}_{4}-l_{5} \dot{\Theta}_{5}=0, \\
\nabla \mathcal{C}_{1}^{\star}: & -b_{1} \dot{\Theta}_{1} \Theta_{1}^{-2} e^{i \gamma_{1}}-l_{8} \dot{\Theta}_{8} \Theta_{8}^{-2}+l_{4} \dot{\Theta}_{4} \Theta_{4}^{-2}+l_{7} \dot{\Theta}_{5} \Theta_{5}^{-2} e^{-i \eta_{1}}=0, \\
\nabla \mathcal{C}_{2}^{\star}: & -l_{1} \dot{\Theta}_{1} \Theta_{1}^{-2}-b_{4} \dot{\Theta}_{2} \Theta_{2}^{-2} e^{i \beta_{1}}-l_{10} \dot{\Theta}_{10} \Theta_{10}^{-2}+l_{9} \dot{\Theta}_{4} \Theta_{4}^{-2} e^{-i \epsilon_{1}}=0, \\
\nabla \mathcal{C}_{3}^{\star}: & -l_{1} \dot{\Theta}_{1} \Theta_{1}^{-2}-l_{2} \dot{\Theta}_{2} \Theta_{2}^{-2}-l_{3} \dot{\Theta}_{3} \Theta_{3}^{-2}+l_{4} \dot{\Theta}_{4} \Theta_{4}^{-2}+l_{5} \dot{\Theta}_{5} \Theta_{5}^{-2}=0 .
\end{array}
$$

Factor out the derivative vector $\dot{\vec{\Theta}}=\left(\dot{\Theta}_{2}, \dot{\Theta}_{3}, \dot{\Theta}_{4}, \dot{\Theta}_{5}, \dot{\Theta}_{8}, \dot{\Theta}_{10}\right)$ and rearrange to obtain

$$
[\nabla \mathcal{C}(\vec{\Theta})] \dot{\vec{\Theta}}=\dot{\Theta}_{1} \vec{b}
$$

where $\vec{b}$ is a vector that is a function of the link dimensions and the various joint angles $\Theta_{i}$. The joint rates can now be obtained by solving (7) for a given input joint rate $\dot{\Theta}_{1}$.

Similarly, to determine the angular joint acceleration, we formulate the second derivative of the loop equations (4) and (5),

$$
\begin{aligned}
\nabla^{2} \mathcal{C}_{1}: & b_{1} \ddot{\Theta}_{1} e^{-i \gamma_{1}}+l_{8} \ddot{\Theta}_{8}-l_{4} \ddot{\Theta}_{4}-l_{7} \ddot{\Theta}_{5} e^{i \eta_{1}}=0 \\
\nabla^{2} \mathcal{C}_{2}: & l_{1} \ddot{\Theta}_{1}+b_{4} \ddot{\Theta}_{2} e^{-i \beta_{1}}+l_{10} \ddot{\Theta}_{10}-l_{9} \ddot{\Theta}_{4} e^{i \epsilon_{1}}=0 \\
\nabla^{2} \mathcal{C}_{3}: & l_{1} \ddot{\Theta}_{1}+l_{2} \ddot{\Theta}_{2}+l_{3} \ddot{\Theta}_{3}-l_{4} \ddot{\Theta}_{4}-l_{5} \ddot{\Theta}_{5}=0 \\
\nabla^{2} \mathcal{C}_{1}^{\star}: & -b_{1}\left(\ddot{\Theta}_{1} \Theta_{1}^{-2}-2 \dot{\Theta}_{1}^{2} \Theta_{1}^{-3}\right) e^{i \gamma_{1}}-l_{8}\left(\ddot{\Theta}_{8} \Theta_{8}^{-2}-2 \dot{\Theta}_{8}^{2} \Theta_{8}^{-3}\right) \\
& +l_{4}\left(\ddot{\Theta}_{4} \Theta_{4}^{-2}-2 \dot{\Theta}_{4}^{2} \Theta_{4}^{-3}\right)+l_{7}\left(\ddot{\Theta}_{5} \Theta_{5}^{-2}-2 \dot{\Theta}_{5}^{2} \Theta_{5}^{-3}\right) e^{-i \eta_{1}}=0 \\
\nabla^{2} \mathcal{C}_{2}^{\star}: \quad & -l_{1}\left(\ddot{\Theta}_{1} \Theta_{1}^{-2}-2 \dot{\Theta}_{1}^{2} \Theta_{1}^{-3}\right)-b_{4}\left(\ddot{\Theta}_{2} \Theta_{2}^{-2}-2 \dot{\Theta}_{2}^{2} \Theta_{2}^{-3}\right) e^{i \beta_{1}} \\
& -l_{10}\left(\ddot{\Theta}_{10} \Theta_{10}^{-2}-2 \dot{\Theta}_{10}^{2} \Theta_{10}^{-3}\right)+l_{9}\left(\ddot{\Theta}_{4} \Theta_{4}^{-2}-2 \dot{\Theta}_{4}^{2} \Theta_{4}^{-3}\right) e^{-i \epsilon_{1}}=0 \\
\nabla^{2} \mathcal{C}_{3}^{\star}: \quad & -l_{1}\left(\ddot{\Theta}_{1} \Theta_{1}^{-2}-2 \dot{\Theta}_{1}^{2} \Theta_{1}^{-3}\right)-l_{2}\left(\ddot{\Theta}_{2} \Theta_{2}^{-2}-2 \dot{\Theta}_{2}^{2} \Theta_{2}^{-3}\right)-l_{3}\left(\ddot{\Theta}_{3} \Theta_{3}^{-2}-2 \dot{\Theta}_{3}^{2} \Theta_{3}^{-3}\right) \\
& +l_{4}\left(\ddot{\Theta}_{4} \Theta_{4}^{-2}-2 \dot{\Theta}_{4}^{2} \Theta_{4}^{-3}\right)+l_{5}\left(\ddot{\Theta}_{5} \Theta_{5}^{-2}-2 \dot{\Theta}_{5}^{2} \Theta_{5}^{-3}\right)=0 .
\end{aligned}
$$

and factor out its second derivative vector $\ddot{\vec{\Theta}}=\left(\ddot{\Theta}_{2}, \ddot{\Theta}_{3}, \ddot{\Theta}_{4}, \ddot{\Theta}_{5}, \ddot{\Theta}_{8}, \ddot{\Theta}_{10}\right)$ to yield

$$
\left[\nabla^{2} \mathcal{C}(\vec{\Theta})\right] \ddot{\vec{\Theta}}=\ddot{\Theta}_{1} \vec{c}+\vec{d},
$$


where $\vec{c}$ and $\vec{d}$ are vectors related to the link dimensions, and the various joint angles $\Theta_{i}$ and rates $\dot{\Theta}_{i}$. Finally, to complete the analysis, equation (9) is solved for the angular joint acceleration for a given input joint acceleration $\ddot{\Theta}_{1}$.

\section{Application: Design of a One Degree of Freedom Mechanical Index Finger based on Anthropometric Backbone Chain and Physiolog- ical Contact Task}

To illustrate the design method presented above, a challenging task of precision grasping of a small object with high curvature is chosen. We start by extracting experimental data from a subject performing a pen grasping task using our developed sensor glove and motion capture system. During each trial, the subject is required to grasps a pen within their reach from a sitting position. Figure 5 shows the index finger moving through the obtained physiological task trajectory between a specified initial (higher) and grasp (lower) locations.

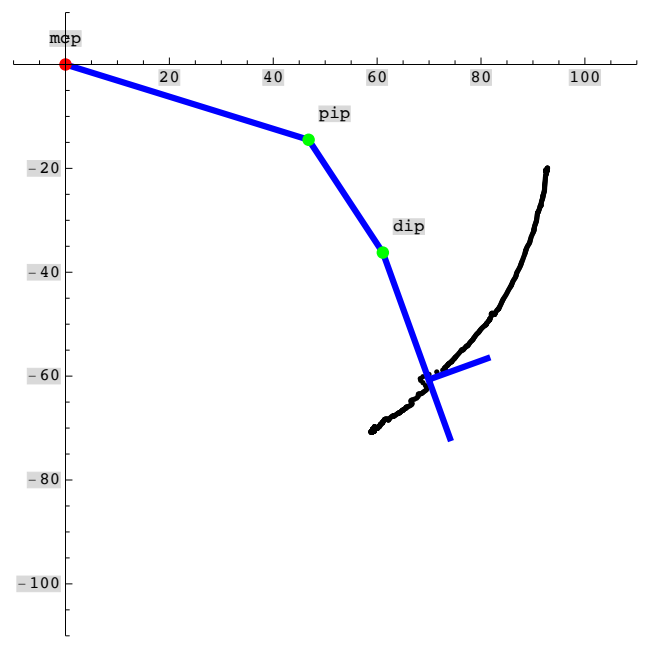

Figure 5: The trajectory of the subject's index finger, moving between the start and the end/grasp positions, obtained from the motion capture system. 


\subsection{Task Specification}

Apart of the two critical poses along the measured trajectory, where the local motions aspect is very important for the finger performance, a velocity at the first pose was defined to keep the finger moving as tangent as possible to the original physiological task trajectory in the vicinity of the specified pose. The grasp position was defined at the point of contact between the finger tip and the pen (lower location in Figure 5), where an acceleration was also specified. Note that in [34], Robson and McCarthy show how these velocity and acceleration specifications are compatible with contact and curvature constraints between the limb and an object. Table 1 shows the task for the trajectory of the tip of the index finger, with an attached moving frame, at each of the two selected poses. They are parametrized in terms of the frame orientation $\theta$, angular velocity $\dot{\theta}$, angular acceleration $\ddot{\theta}$, as well as the frame origin $d_{x}$ and $d_{y}$, velocity $\dot{d}_{x}$ and $\dot{d}_{y}$, and acceleration $\ddot{d}_{x}$ and $\ddot{d}_{y}$.

Table 1: The physiological task data, consisting of two positions $\mathrm{P}$, two velocities $\mathrm{V}$ and one acceleration A defined in the second position compatible with the object local curvature. The task specifications are experimentally obtained from the movement of the tip of the subject's index finger.

\begin{tabular}{lcc}
\hline Task & 1 & 2 \\
\hline$P:\left(\theta^{\circ}, d_{x} m m, d_{y} m m\right)$ & $(-35.9,92.8,-20.0)$ & $(-78.7,61.4,-68.5)$ \\
$V:\left(\dot{\theta}^{\circ} / s, \dot{d}_{x} m m / s, \dot{d}_{y} m m / s\right)$ & $(-2.8,-0.7,-3.3)$ & $(4.9,-6.3,-5.0)$ \\
$A:\left(\ddot{\theta}^{\circ} / s^{2}, \ddot{d}_{x} m m / s^{2}, \ddot{d}_{y} m m / s^{2}\right)$ & - & $(-1252,-496,-336)$ \\
\hline
\end{tabular}

\subsection{Backbone Chain Position, Velocity and Acceleration Inverse Kinematics}

For the two task poses, we can identify the position, velocity and acceleration of each of the links of the parallel 3R robot using the equations defined in Eq. (2). The inverse kinematic solutions for the two selected poses are as shown in Table 2. 
Table 2: Inverse kinematics solution of the chosen parallel 3R backbone chain at each of the tasks positions.

\begin{tabular}{ccccccc}
\hline Task & $\theta_{1}\left(^{\circ}\right)$ & $\theta_{2}\left(^{\circ}\right)$ & $\theta_{3}\left({ }^{\circ}\right)$ & $\theta_{4}\left({ }^{\circ}\right)$ & $\theta_{5}\left({ }^{\circ}\right)$ & $\theta_{6}\left(^{\circ}\right)$ \\
\hline 1 & 8.18 & -34.98 & -9.08 & -67.62 & -63.58 & 5.32 \\
2 & -23.55 & -40.56 & -14.58 & -116.18 & -29.00 & -23.51 \\
\hline \hline Task & $\dot{\theta}_{1}\left({ }^{\circ} / s\right)$ & $\dot{\theta}_{2}\left({ }^{\circ} / s\right)$ & $\dot{\theta}_{3}\left({ }^{\circ} / s\right)$ & $\dot{\theta}_{4}\left({ }^{\circ} / s\right)$ & $\dot{\theta}_{5}\left({ }^{\circ} / s\right)$ & $\dot{\theta}_{6}\left({ }^{\circ} / s\right)$ \\
\hline 1 & -2.11 & 0.94 & -1.66 & -2.95 & 2.30 & -2.17 \\
2 & -2.05 & -17.10 & 24.03 & 3.80 & -18.53 & 19.62 \\
\hline \hline Task & $\ddot{\theta}_{1}\left({ }^{\circ} / s^{2}\right)$ & $\ddot{\theta}_{2}\left({ }^{\circ} / s^{2}\right)$ & $\ddot{\theta}_{3}\left({ }^{\circ} / s^{2}\right)$ & $\ddot{\theta}_{4}\left(\circ / s^{2}\right)$ & $\ddot{\theta}_{5}\left({ }^{\circ} / s^{2}\right)$ & $\ddot{\theta}_{6}\left({ }^{\circ} / s^{2}\right)$ \\
\hline 2 & -417.33 & 920.99 & -1755.84 & -1250.61 & 2092.77 & -2094.33 \\
\hline
\end{tabular}

Table 3: Solution for the two chosen RR links $\mathbf{G}_{i} \mathbf{W}_{i}$

\begin{tabular}{ccc}
\hline Solution & $\mathbf{G}_{i}^{1}$ & $\mathbf{W}_{i}^{1}$ \\
\hline 1 & $(109.92,-1.33)$ & $(94.17,6.94)$ \\
\hline 2 & $(-62.48,78.59)$ & $(-51.42,73.36)$ \\
\hline
\end{tabular}

\subsection{Geometric Design of a Wearable Device for an Index Finger}

A search through all seventeen different topologies shown in Figure 3 led to a particular linkage which was both compact and easy to manufacture. The solutions are listed in Table 3. The kinematic structure and synthesis results for this particular eight-bar linkage are shown in Figure 6. Kinematic analysis was performed to ensure that the linkage is moving smoothly throughout the specified second order constraint task without any circuit or branch defects.

\subsection{Prototype Development and Validation}

Figure 7 shows the CAD drawing of the chosen one degree-of-freedom eight bar wearable device. Figure 8 shows the 3D printed prototype in which the anthropometric 3R backbone parallels the human's index finger and is attached to it using velcro straps. This wearable device, based on physiological contact 


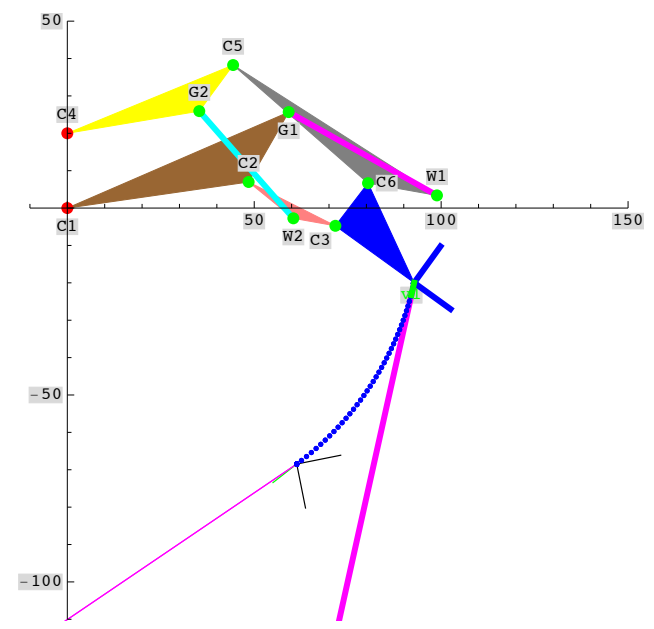

Figure 6: The topology chosen and the trajectory of the synthesized eight-bar linkage.

task with second order motion constraints, is currently passively actuated by the flexion/extension degrees of freedom of the metacarpophalangeal (MCP) joint. To test the prototype performance and identify the effects this wearable device have on the movement of the index finger, the kinematic trajectories of the tip of the index finger with and without the device were recorded using motion capture system. During each capture session, the same subject, described in the previous section, sits at the table such that their arm rests on the table in a relaxed position with a pen placed within their reach, and when prompted grasps it.

Figure 9 shows a comparison between the anthropomorphic trajectory, obtained from the motion capture system and the trajectory of the synthesized index finger. It can be seen that in general the finger follows closely the task trajectory throughout the experimentally obtained physiological path with an overall root-mean-square (RMS) error of $0.82 \mathrm{~mm}$. It can also be noticed that due to the higher order motion specifications defined in the start and end pose, related to contact and curvature requirements for the fingers motion through the physiological path, the two trajectories overlap in the vicinity of both critical locations. 


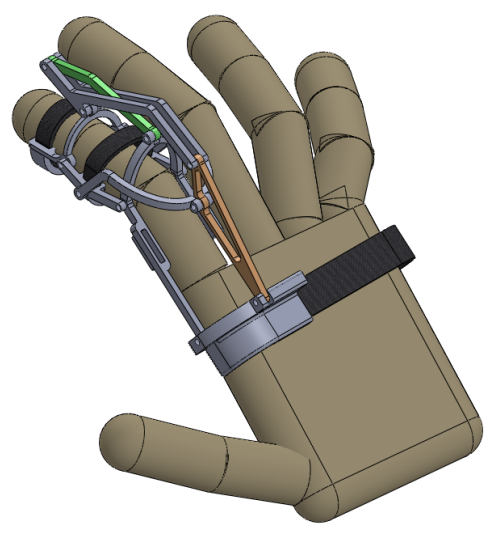

Figure 7: CAD drawing of the one degree of freedom wearable device with the $3 \mathrm{R}$ anthropometric backbone chain replaced by the human's finger.

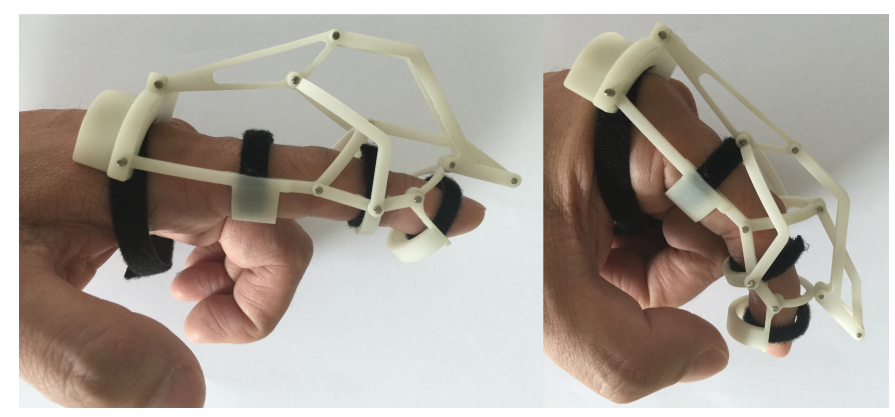

Figure 8: Prototype of the one degree of freedom skeletal structure, based on anthropometric backbone chain and second-order motion physiological contact task.

\section{Conclusions}

This paper describes a systematic method for the kinematic synthesis of onedegree-of-freedom mechanical wearable devices that incorporate multi-loop linkage based structures and second order task specifications, resulting in minimally actuated skeletal structures. The method offers a novel alternative approach for wearable device design, that incorporates an anthropometric backbone chain and are attached parallel to the human's limb. The paper focuses on grasping task and discusses how to use sensor-based task data to formulate the physiological contact task and the synthesis equations for the design of the limb of a 


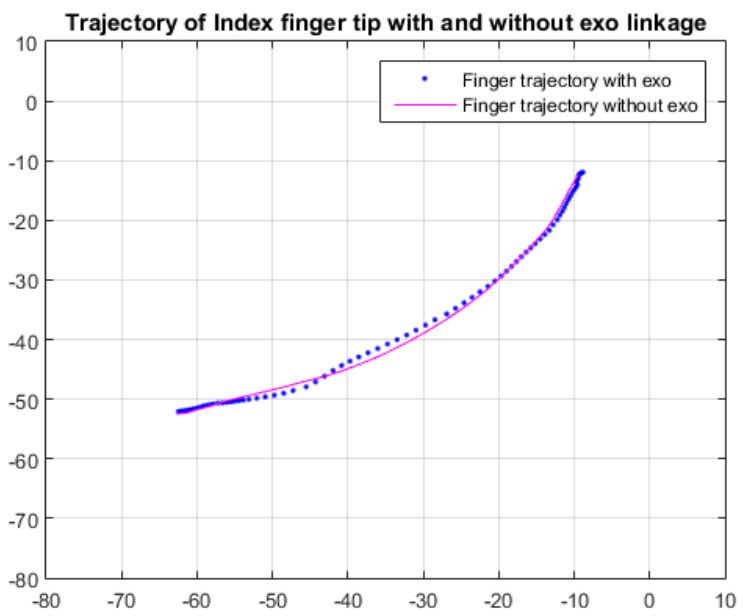

Figure 9: Motion trajectories of the index finger tip with and without the exoskeleton.

wearable grasping device. The combination of second order motion task specifications and anthropometric back-bone chain is a novel approach, which provides a solution to some of the open problems in the field, by offering a different alternative technique for one degree-of-freedom skeletal structure designs. The result is mechanical limbs with minimal number of actuators, lower complexity, thus less mass and cost, as well as better wearability.

The addition of requirements on the accelerations of the fingertips allows for the definition of contact physiological tasks, taking into account the local curvature of the contact at the fingertips for grasp stability. The preliminary results presented in this paper are the first step towards developing a novel methodology for the design of minimally actuated multi-loop grasping devices for human-like tasks that involve contact with objects or environment. Future directions include the complete design of a passive multi-finger grasping wearable hand, as well as expanding the developed method to spatial motions. 


\section{Acknowledgement}

The authors gratefully acknowledge the support of NSF grant award Id: 1404011 and NSF grant sub-award Id: 2013-2908, as well as SUTD Start-Up Grant (SRG EPD 2012 025), ZJU-SUTD Research Collaboration Grant (SUTDZJU/RES/03/2013) and Temasek Laboratories@SUTD-STARS (IGDST1301013).

\section{References}

[1] M. Ceccarelli, 2004, Fundamentals of Mechanics of Robotic Manipulation, Dordrecht: Kluwer/Springer.

[2] C. M. Gosselin, T. Laliberte, 1998, "Underactuated Mechanical Finger with Return Actuation". US Patent 5762390.

[3] N. Fukaya, S. Toyama, T. Asfour, R. Dillmann, 2000, "Design of the TUAT/Karlsruhe Humanoid Hand", IEEE/RSJ International Conference on Intelligent Robots and Systems, Takamatsu, pp. 1754-1759.

[4] S. Baek, S.H.Lee, J.Chang, 1999, "Design and Control of Robotic Finger for Prosthetic Hands", Proc. of IEEE/RSJ International Conference on Intelligent Robots and Systems, pp.113-117.

[5] J. F. Veneman, R. Kruidhof, E. E. Hekman, R. Ekkelenkamp, E. H. Van Asseldonk, and H. van der Kooij, 2007, "Design and evaluation of the LOPES exoskeleton robot for interactive gait rehabilitation," Neural Systems and Rehabilitation Engineering, IEEE Transactions on, vol. 15, pp. 379-386.

[6] S. Jezernik, G. Colombo, T. Keller, H. Frueh, and M. Morari, 2003, "Robotic orthosis lokomat: A rehabilitation and research tool," Neuromodulation, vol. 6, pp. 108-115.

[7] R. Gopura and K. Kiguchi, 2009, "Mechanical designs of active upper-limb exoskeleton robots: State-of-the-art and design difficulties," in Rehabilitation Robotics ICORR 2009. IEEE International Conference on, pp. 178187. 
[8] H. S. Lo and S. Q. Xie, 2012, "Exoskeleton robots for upper-limb rehabilitation: State of the art and future prospects" Medical engineering 8 physics, vol. 34, pp. 261-268.

[9] J.Wang, J.Zhang, S.Wang, 2009, "Design of an Exoskeleton for Index Finger Rehabilitation", 31 Annual International Conference of the IEEE EMBS, Minneapolis, Minnesota, pp.5957-5960.

[10] Y. Yihun, R. Miklos, A. Perez, D. Reinkensmeyer, K. Denney, E. Wolbrecht, 2011, "Single Degree-of-Freedom Exoskeleton Mechanism Design for Thumb Rehabilitation", 34 Annual International IEEE EMBS Conference.

[11] M. Brown, N. Tsagarakis, and D. Caldwell, 2003, "Exoskeletons for human force augmentation," Industrial Robot: An International Journal, vol. 30, pp. 592-602.

[12] H. Kazerooni, "Exoskeletons for human power augmentation," 2005, in Intelligent Robots and Systems, IEEE/RSJ International Conference on, pp. 3459-3464.

[13] A. B. Zoss, H. Kazerooni, and A. Chu, 2006, "Biomechanical design of the Berkeley lower extremity exoskeleton (BLEEX)," Mechatronics, IEEE/ASME Transactions on, vol. 11, pp. 128-138.

[14] J. C. Perry, J. Rosen, and S. Burns, 2007, "Upper-limb powered exoskeleton design," Mechatronics, IEEE/ASME Transactions on, vol. 12, pp. 408-417.

[15] P. Heo, G. M. Gu, S. Lee, K. Rhee and J. Kim, 2007, "Current Hand Exoskeleton Technologies for Rehabilitation and Assistive Engineering," International Journal of Precision Engineering and Manufacturing, 13(5):807-824.

[16] Worsnopp, T. T., Peshkin, M. A., Colgate, J. E. and Kamper, D. G., 2007, "An Actuated Finger Exoskeleton for Hand Rehabilitation Follow- 
ing Stroke, Proc. of the IEEE International Conference on Rehabilitation Robotics, pp. 896-901.

[17] Fontana, M., Dettori, A., Salsedo, F. and Bergamasco, M., 2009, "Mechanical design of a novel Hand Exoskeleton for accurate force displaying, Proc. of the IEEE International Conference on Robotics and Automation, pp. 1704-1709.

[18] Nakagawara, S., Kajimoto, H., Kawakami, N., Tachi, S. and Kawabuchi, I., 2005, "An Encounter-Type Multi-Fingered Master Hand Using Circuitous Joints, Proc. of the IEEE International Conference on Robotics and Automation, pp. 2667-2672.

[19] Wege, A. and Hommel, G., 2005, "Development and control of a hand exoskeleton for rehabilitation of hand injuries, Proc. of the IEEE/RSJ International Conference on Intelligent Robots and Systems, pp. 3046-3051.

[20] In, H. K., Cho, K.-J., Kim, K. R. and Lee, B. S., 2011, "Jointless structure and under-actuation mechanism for compact hand exoskeleton, Proc. of the IEEE International Conference on Rehabilitation Robotics, pp. 1-6.

[21] In, H. K. and Cho, K. J., 2009, "Compact Hand Exoskeleton Robot for the Disabled, Proc. of the International Conference on Ubiquitous Robots and Ambient Intelligence.

[22] Kadowaki, Y., Noritsugu, T., Takaiwa, M., Sasaki, D. and Kato, M., 2011, "Development of Soft Power-Assist Glove and Control Based on Human Intent, Journal of Robotics and Mechatronics, Vol. 23, No. 2, pp. 281-291.

[23] Stergiopoulos, P., Fuchs, P. and Laurgeau, C., 2003, "Design of a 2- finger hand exoskeleton for VR grasping simulation, Proc. of the Eurohaptics, pp. 80-93.

[24] N. P. Robson, J. Allington and G. S. Soh, 2014, "Development Of Underactuated Mechanical Fingers Based On Anthropometric Data And An- 
thropomorphic Tasks", ASME International Design Engineering Technical Conference, Buffalo, NY.

[25] F. Sergi, D. Accoto, N. L. Tagliamonte, G. Carpino, E. Guglielmelli, 2011, "A systematic graph-based method for the kinematic synthesis of nonanthropomorphic wearable robots for the lower limbs, Frontiers of Mechanical Engineering, vol. 6, no. 1, pp. 61-70.

[26] D. Accoto, F. Sergi, N. L. Tagliamonte, G. Carpino, A. Sudano, E. Guglielmelli, 2014, "Robomorphism: a nonanthropomorphic wearable robot, IEEE Robotics and Automation Magazine, vol. 21(4), pp 45-55.

[27] P. Agarwal, A. Hechanova, and A. D. Deshpande, 2013, "Kinematics and dynamics of a biologically inspired index finger exoskeleton, in ASME Dynamic Systems and Control Conference.

[28] W. S. Howard and V. Kumar, 1996, "On the Stability of Grasped Objects," IEEE Transaction of Robotics and Automation, vol. 12(6), pp 904-917.

[29] E. Rimon and J. Burdick, 1998, ?Mobility of Bodies in Contact?Part I: A 2nd-Order Mobility Index for Multiple-Finger Grasps,? IEEE Transaction of Robotics and Automation, vol. 14(5), pp 696-708.

[30] E. Rimon and J. Burdick, 1998, ?Mobility of Bodies in Contact?Part II: How Forces are Generated by Curvature Effects,? IEEE Transaction on Robotics and Automation, vol. 14(5), pp 709-717.

[31] S. Ghosh, H.S. Moon, N. Robson, 2013, "Reconstructing Humans Hand Motion: Preliminary Results and Applications in the Design of Mechanical Fingers for Anthropomorphic Tasks, ASME International Design Engineering Technical Conference, Portland, OR.

[32] N. Robson, S. Ghosh, G.S. Soh, 2013, "Development of a Sensor-Based Glove Device for Extracting Human Finger Motion Data used in the Design of Minimally-Actuated Mechanical Fingers", 3-rd IFToMM International Symposium on Robotics and Mechatronics, Oct. 24, Singapore. 
[33] J. M. McCarthy and G.S. Soh, 2010, Geometric Design of Linkages, Second Edition, Interdisciplinary Applied Mathematics, Springer Verlag, New York.

[34] Robson N. and J. M. McCarthy, 2007, "Kinematic Synthesis with Contact Direction and Curvature Constraints on the Workpiece", Proc. ASME International Design Engineering Technical Conferences, Las Vegas, NV, vol. 8, pp. 581-588.

[35] G. S. Soh and N. P. Robson, 2013, "Kinematic Synthesis of Minimally Actuated Multi-Loop Planar Linkages with Second Order Motion Constraints for Object Grasping", ASME Dynamic Systems and Control Conference, Stanford, USA.

[36] C. W. Wampler, 2001, "Solving the Kinematics of Planar Mechanisms by Dixon Determinant and a Complex Plane Formulation", ASME Journal of Mechanical Design, 123(3), pp. 382-387 